\title{
Estudio de la Flexión de una Viga de Material Elástico no Lineal
}

\author{
Tarsicio Beléndez ${ }^{1}$, Cristian Neipp ${ }^{2}$ y Augusto Beléndez ${ }^{2}$ \\ ${ }^{1}$ Departamento de Ciencia y Tecnología de los Materiales \\ Universidad Miguel Hernández de Elche \\ Avda. del Ferrocarril, s/n. E-03202. Elche (Alicante). España \\ ${ }^{2}$ Departamento de Física, Ingeniería de Sistemas y Teoría de la Señal \\ Universidad de Alicante. Apartado 99. E-03080 Alicante. España
}

Recebido em 31 de janeiro, 2002. Aceito em 13 de agosto, 2002.

\begin{abstract}
En los cursos de Física y Mecánica para estudiantes universitarios de Ciencias e Ingeniería se estudian los sólidos deformables. Cuando un sólido se somete a un esfuerzo, sufre una deformación que puede llegar incluso, cuando el esfuerzo es considerable, a la destrucción del sistema. Si el sólido recupera su forma original después de suprimir las fuerzas externas, se dice que la deformación ha sido elástica y si, además, la deformación producida es proporcional a la tensión aplicada entonces se habla de sólido elástico lineal. Una de las deformaciones que puede sufrir un sólido es la flexión. Cuando una barra delgada o una viga empotrada en una pared se somete a un esfuerzo de flexión, la barra se deforma curvándose. También en este caso en los primeros cursos universitarios de las materias antes señaladas se analiza la flexión de una viga supondiendo que el material del que está fabricada es elástico y lineal y así podemos encontralo en los textos de Física y Mecánica de Sólidos. Sin embargo, cada vez más se utilizan materiales cuyo comportamiento es no lineal y éste hecho debería considerarse. En este trabajo se analiza la flexión de una viga supondiendo que el material de la misma es no lineal. Se obtienen las ecuaciones de la elástica y la flecha siguiendo un tratamiento análogo al desarrollado por Feynman cuando estudia la viga flexionada en su libro de Física, por lo que el desarrollo aquí presentado podría ser seguido sin muchos problemas por cualquier estudiante de Física de los primeros cursos universitarios.
\end{abstract}

\section{Introducción}

Las vigas generalmente son cuerpos sólidos de forma alargada y sección recta constante, de gran interés en ingeniería y arquitectura, que normalmente se utilizan en posición horizontal y siendo su longitud grande comparada con las dimensiones de su sección recta. Las vigas pueden estar sometidas a cargas concentradas, cargas distribuidas o a pares (momentos concentrados) que actúen solos o en una combinación cualquiera, siendo la flexión la principal deformación que sufren [1].

Puede definirse una viga como un sólido homogéneo e isótropo engendrado por una sección transversal, que generalmente admite un plano de simetría y cuyo centro de gravedad describe una curva o línea, denominada directriz, siendo el plano que contiene a la sección transversal normal a dicha directriz [2]. Un caso particular de vigas que se estudian en los primeros cursos universitarios de Física de las carreras de Ingeniería y Arquitectura son las estáticamente determinadas o isostáticas $[1,2]$, para las que se pueden obtener las reacciones de los apoyos a partir de las ecuaciones de la Estática, es decir, imponiendo las condiciones de que la suma de fuerzas sea nula y la suma de momentos respecto a un punto también lo sea. El estudio de la flexión de las vigas isostáticas puede encontrarse en algunos textos de Física General y Mecánica (en su parte de Estática) $[1,4]$ y Mecánica de Materiales [5,6]. Asimismo, las vigas en voladizo han recibido recientemente atención en revistas en las que se publican trabajos relacionados con la enseñanza de la Física a nivel universitario [7-10]. Sin embargo, para el análisis que vamos a considerar en este trabajo basta con el estudio de la viga flexionada que hace Feynman [11], al y suficiente para comprender el comportamiento de estos elementos constructivos cuando se someten a acciones externas.

Muchos problemas en Mecánica de Sólidos pueden resolverse utilizando el análisis lineal [6]. Sin embargo, esta situación cambia cuando se trata de plantear y solucionar problemas no lineales. Entre las causas de no linealidad en Mecánica de Sólidos se encuentran la no linealidad geométrica, la no linealidad debida al comportamiento del material y la no linealidad debida a las condiciones de contorno [12]. En todos los casos la so- 
lución analítica suele ser complicada o inexistente salvo en situaciones sencillas, por lo que es necesario recurrir a técnicas de solución mediante métodos numéricos. En los libros de Física General y Mecánica y en los textos elementales de Mecánica de Sólidos se estudian únicamente problemas cuya solución puede encontrarse bajo la hipótesis del análisis lineal, mientras que los problemas no lineales apenas se analizan, aún cuando hay muchas situaciones reales bastante comunes que son claramente no lineales [10].

En el presente trabajo se plantea una forma sencilla de introducir a los estudiantes de los primeros cursos universitarios el concepto de no linealidad debida al comportamiento del material. Para ello se analiza la flexión de una viga en voladizo en el caso particular en que el material del que está fabricada es elástico no lineal, a diferencia del caso lineal que es el que se estudia en todos los libros de texto antes señalados. El desarrollo que se va a realizar es análogo al presentado por Feynman en su libro [11] y material elástico no lineal que se va a considerar es el conocido como de tipo Ludwick $[12,13]$ pues presenta la ventaja que incluye al material elástico lineal como caso particular, junto con un tratamiento matemático sencillo. Esto es didácticamente muy interesante pues permite a los estudiantes derivar las ecuaciones que le son más familiares correspondientes al material elástico lineal de las más generales del material elástico no lineal de tipo Ludwick.

Se van a obtener dos características de la viga: el desplazamiento máximo del extremo libre de la barra o flecha en función de la fuerza aplicada y la curva deformada que adopta la barra flexionada y que se denomina elástica [11], en el caso de una viga empotrada en un extremo, también denominada viga en voladizo o ménsula. Tanto la flecha como la elástica dependen del material y de la geometría de la viga $[2,3]$. Se estudiarán algunas situaciones particulares en las que se puede obtener la ecuación de la elástica por integración inmediata de la ecuación diferencial que gobierna el comportamiento de la viga.

\section{Ecuación momento-curvatura para una viga de material elástico no lineal}

Consideremos una viga de sección rectangular constante sometida a una serie de cargas externas que la deforman. Si la barra está curvada significa que el material de la parte interna de la curva está comprimido, mientras que el material de la parte externa está estirado como se muestra en la Fig. 1 (a). Existe una superficie que no está ni comprimida ni estirada y que se conoce como superficie neutra $[2,6]$. Consideraremos tres hipótesis relativas a la forma en que el esfuerzo de- forma al material de la viga [6]. Para pequeñas flexiones de vigas simples el eje longitudinal de la viga, es decir, la línea que une los centros de gravedad de las secciones transversales de la viga, no experimenta ningún cambio de longitud y se conoce como línea neutra. Es evidente que la línea neutra pertenece a la superficie neutra de la viga. El momento tiende a deformar la viga en forma tal que esta línea recta se vuelve una línea curva. La segunda hipótesis es que todas las secciones transversales de la viga permanecen planas y perpendiculares al eje longitudinal durante la deformación. La tercera hipótesis es que cualquier deformación de la sección transversal dentro de su propio plano será despreciada. En particular, el eje contenido en el plano de la sección tranversal y respecto al cual gira la sección se llama eje neutro.

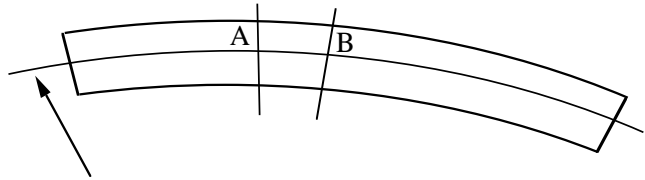

superficie neutra

(a)

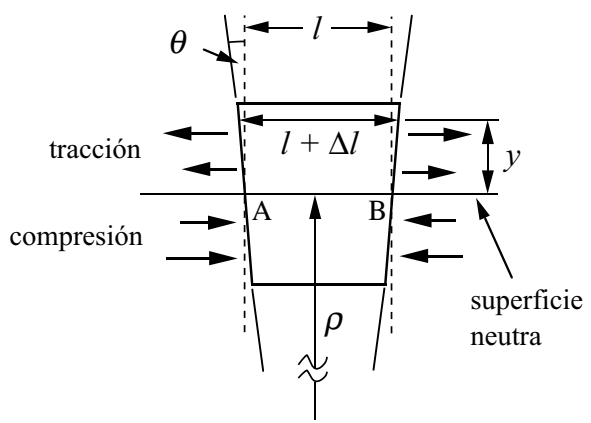

(b)

Figura 1. (a) Viga flexionada y (b) segmento pequeño de la viga flexionada.

Si el comportamiento del material es lineal, la ley de Bernoulli-Euler [6, 11] indica que el momento flector es inversamente proporcional al radio de curvatura, sin embargo si el comportamiento del material no es lineal esto so se cumple. Para flexión pura, una rebanada delgada de la barra se deforma como se ve en la Fig. 1 (b). El material por debajo de la superficie neutra tiene una deformación compresional, que veremos es proporcional a la distancia a la superficie neutra, mientras que el material por encima está estirado, también en proporción a la distancia a la superficie neutra. De la Fig. 1 (b) es fácil ver que la relación entre el estiramiento longitudinal $\Delta l$ de una fibra de la viga, la altura $y$ (Fig. 2) y longitud de la fibra antes de la deformación $l$ y el radio de curvatura $\rho$, es $2 \theta=\Delta l / y=l / \rho$, de donde:

$$
\frac{\Delta l}{l}=\frac{y}{\rho}
$$




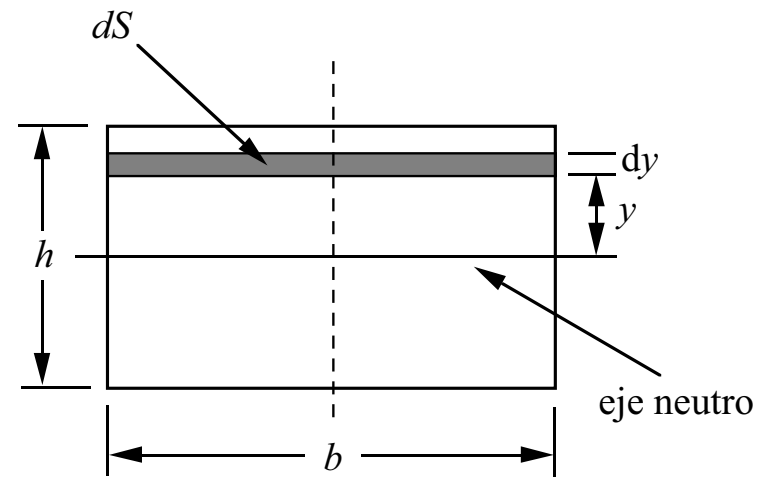

Figura 2. Sección transversal de la viga.

Si suponemos que el material es elástico no lineal de tipo Ludwick, la relación entre la fuerza por unidad de área (el esfuerzo $\sigma=d F / d S$ ) y el alargamiento por unidad de longitud de una fibra cualquiera (la deformación $\varepsilon=\Delta l / l)$ se escribe en la forma $[12,13]$ :

$$
\sigma=E \varepsilon^{1 / n}
$$

donde $E$ y $n$ son constantes características que dependen del material. La relación tensión-deformación no lineal de tipo Ludwick de la ecuación (2) se ha representado en la Fig. 3 para distintos valores de $n$, incluyendo el caso elástico lineal $n=1$, mientras que en la Tabla I se presentan los valores de $E$ y $n$ para algunos materiales de tipo Ludwick [6, 12].

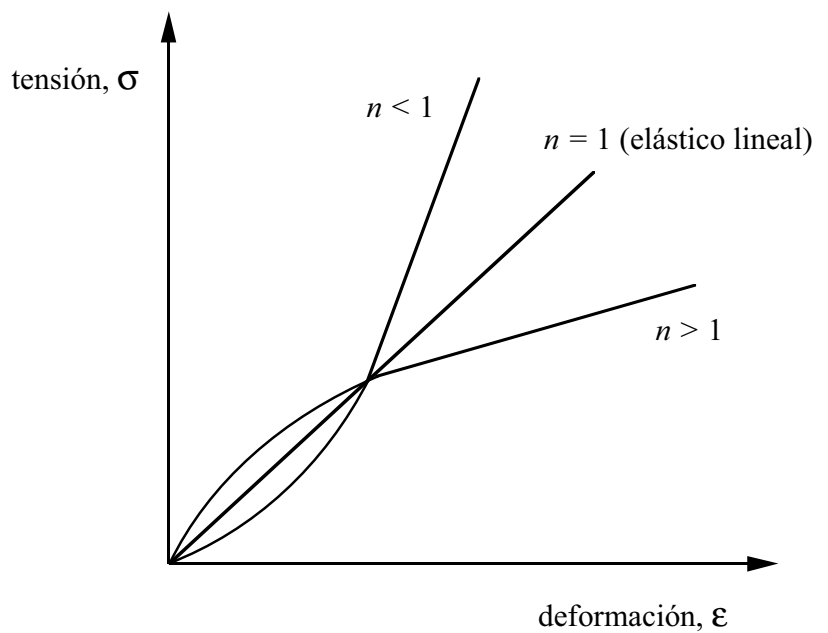

Figura 3. Relación tensión-deformación no lineal de tipo Ludwick para distintos valores de $n$, incluyendo el caso elástico lineal $n=1$.
TABLA I - Valores de $E$ y $n$ para distintos materiales de tipo Ludwick

\begin{tabular}{lcc}
\hline MATERIAL & $\boldsymbol{E}$ (MPa) & $\boldsymbol{n}$ \\
\hline Aleación de aluminio N.P.8 & 456 & 4.79 \\
\hline Cobre endurecido & 459 & 2.16 \\
\hline Fibra de vidrio & 300 & 2.00 \\
\hline Plástico acetal & 66 & 3.00 \\
\hline Plástico polipropileno & 10 & 4.00 \\
\hline
\end{tabular}

Podemos escribir la ecuación (2) en la forma:

$$
\frac{d F}{d S}=E\left(\frac{\Delta l}{l}\right)^{1 / n}
$$

Haciendo uso de las ecuaciones (1) y (3) es posible calcular la fuerza que actúa sobre una superficie $d S$ situada a una distancia $y$ de la superficie neutra (Fig. 2), cuya expresión es:

$$
d F=E\left(\frac{y}{\rho}\right)^{1 / n} d S
$$

Es evidente de la ecuación (4) que $n=1$ corresponde al caso elástico lineal y entonces la constante $E$ es el módulo de Young del material, de modo en este caso la ecuación (4) coincide con la ecuación $38.34 \mathrm{del}$ libro de Feynman [11].

Veamos a continuación cuáles son las fuerzas que dan lugar a tales deformaciones. Las fuerzas que actúan sobre el pequeño segmento dibujado en la Fig. 1 (b) están dibujadas en la misma. Si consideramos cualquier corte transversal, las fuerzas actúan a través de él, en un sentido por encima de la superficie neutra y en el otro por debajo. Vienen en pares para dar lugar a lo que se conoce como "momento flector", $M$, que representa el momento de las fuerzas respecto a la línea neutra. Es posible calcular el momento total integrando la fuerza multiplicada por la distancia a la supeficie neutra (Fig. 2) en una de las caras del segmento de la Fig. 1 (b):

$$
M=\int_{S} y d F
$$

donde la integral se extiende a la sección transversal $S$ de la barra.

Haciendo uso de la ecuación (4), el momento flector de la ecuación (5) se puede expresar en la forma:

$$
M=\int_{S} y E\left(\frac{y}{\rho}\right)^{1 / n} d S
$$

que finalmente puede escribirse como:

$$
M=\frac{E}{\rho^{1 / n}} \int_{S} y^{(n+1) / n} d S
$$


La integral de $y^{(n+1) / n} d S$ es el "momento de orden $(n+1) / n$ ", que vamos a denominar $I_{n}$, de la sección transversal respecto a un eje horizontal que pasa por su centro de gravedad:

$$
I_{n}=\int_{S} y^{(n+1) / n} d S
$$

por lo que la ecuación (7) queda finalmente:

$$
M=\frac{E I_{n}}{\rho^{1 / n}}
$$

La ecuación (9) proporciona la relación entre el momento flector $M$ y el radio de curvatura $\rho$ de la viga y se conoce como ecuación momento-curvatura. La "rigidez" de la viga es proporcional al producto $E I_{n}$.

El radio de curvatura $\rho$ de una curva de ecuación $z=z(x)$ (ecuación cartesiana de la curva elástica de la viga) puede calcularse mediante la ecuación $[6,11]$ :

$$
\frac{1}{\rho}=\frac{d^{2} z / d x^{2}}{\left[1+(d z / d x)^{2}\right]^{3 / 2}}
$$

En el caso particular en que el desplazamiento de los puntos de la elástica de la viga deformada respecto a los de la viga sin deformar es pequeño, es decir, en el caso de pendientes pequeñas, es posible despreciar el término $(d z / d x)^{2}$ frente a la unidad en la ecuación (10), por lo que el radio de curvatura se puede calcular a partir de la ecuación:

$$
\frac{1}{\rho}=\frac{d^{2} z}{d x^{2}}
$$

Lo que permite escribir la ecuación diferencial de la curva elástica para una viga de material elástico no lineal de tipo Ludwick bajo la hipótesis de pequeños desplazamientos en la forma:

$$
\frac{d^{2} z}{d x^{2}}=\left(\frac{M}{E I_{n}}\right)^{n}
$$

que es la ecuación diferencial de la elástica de la viga de material de tipo Ludwick en el caso de pequeñas pendientes de la misma.

Si la sección transversal de la viga es rectangular de base $b$ y altura $h$ (Fig. 2), entonces en la ecuación (8) se tiene $d S=b d y$, de donde $I_{n}$ se puede calcular como:

$$
I_{n}=\int_{-h / 2}^{h / 2} y^{(n+1) / n} b d y
$$

cuya integración es inmediata y proporciona el valor:

$$
I_{n}=\left(\frac{1}{2}\right)^{(n+1) / n}\left(\frac{n}{2 n+1}\right) b h^{(2 n+1) / n}
$$

que para el caso de comportamiento lineal del material $(n=1)$ corresponde al momento de inercia $I_{1}$ de la sección respecto al eje perpendicular a la misma (eje neutro) y que pasa por su centro de gravedad:

$$
I_{1}=\frac{1}{12} b h^{3}
$$

Por otra parte, es posible calcular las tensiones que se ejercen sobre las distintas fibras de la viga. De las ecuaciones (1) y (9), teniendo en cuenta que $\varepsilon=\Delta l / l$ $=y / \rho$, queda:

$$
\sigma=\frac{M y^{1 / n}}{I_{n}}
$$

\section{Elástica y flecha para una viga en voladizo}

Para obtener la ecuación de la curva elástica $z=z(x)$ es necesario conocer el momento flector $M$. Como ejemplo consideremos una viga empotrada en un extremo y libre en el otro, también conocida como viga en voladizo o ménsula, sobre la que se aplica una fuerza concentrada $F$ en el extremo libre y una fuerza $P$ uniformemente distribuida a lo largo de la longitud de la viga (que puede ser, por ejemplo, el peso de la propia viga). La Fig. 4 muestra una viga en voladizo de longitud $L$, sección rectangular constante, peso $P$ uniformemente repartido a lo largo de su longitud y que soporta una carga concentrada $F$ en el extremo libre. El momento flector es una función de la coordenada $x$ ya que es el momento con respecto al eje neutro de cualquier sección. Para la viga en voladizo considerada, el momento flector $M_{F}$ debido a la carga puntual Faplicada en el extremo de la viga respecto a la sección situada a una distancia $x$ del empotramiento puede calcularse fácilmente mediante la ecuación $[7,11]$ :

$$
M_{F}(x)=F(L-x)
$$

mientras que el momento flector debido a la carga uniformemente distribuida a lo largo de la longitud de la viga tiene la expresión $[2,7]$ :

$$
M_{P}(x)=\frac{P}{2 L}(L-x)^{2}
$$

El momento flector total $M(x)$ será la suma de los dos anteriores, es decir,

$$
M(x)=F(L-x)+\frac{P}{2 L}(L-x)^{2}
$$

Sustituyendo en la ecuación diferencial de la elástica (12) para pequeños desplazamientos (pequeñas pendientes), ésta toma la forma:

$$
\frac{d^{2} z}{d x^{2}}=\frac{1}{\left(E I_{n}\right)^{n}}\left[F(L-x)+\frac{P}{2 L}(L-x)^{2}\right]^{n}
$$

que puede escribirse también como: 


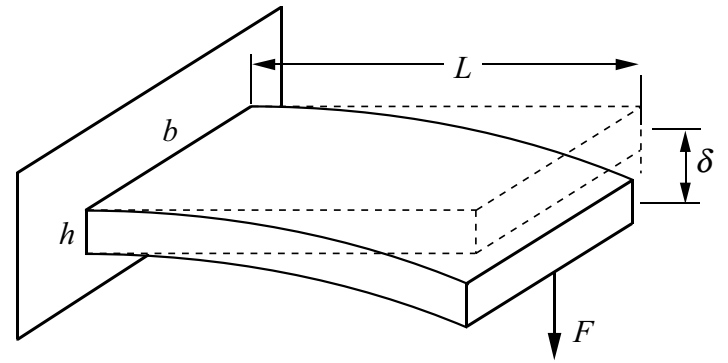

(a)

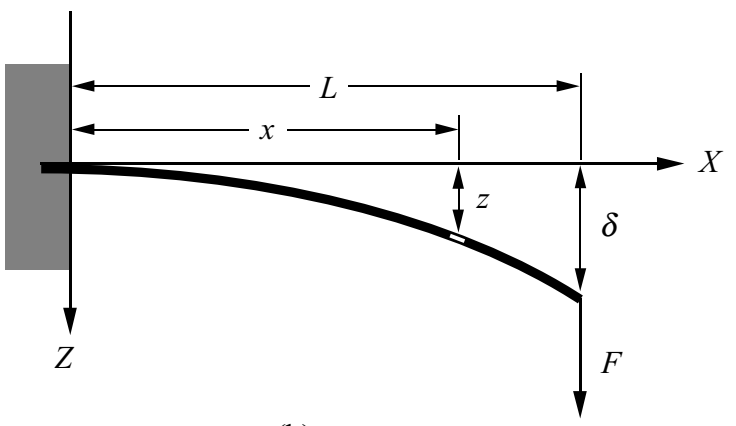

(b)

Figura 4. (a) Flexión de una viga en voladizo de sección rectangular cuando se aplica uuna carga concentrada en el extremo libre. (b) Elástica de la viga en voladizo.

$$
\frac{d^{2} z}{d x^{2}}=\left(\frac{F}{E I_{n}}\right)^{n}(L-x)^{n}\left[1+\frac{P}{2 F L}(L-x)\right]^{n}
$$

donde para la viga en voladizo las condiciones de contorno son:

$$
\begin{gathered}
z(0)=0 \\
\left(\frac{d z}{d x}\right)_{x=0}=0
\end{gathered}
$$

En general la ecuación (20) es una ecuación diferencia de segundo orden no lineal que debe resolverse numéricamente. Sin embargo, es posible considerar algunos casos particulares de gran interés didáctico para los que la integración es inmediata. Veamos a continuación algunos de ellos.

\section{III.1. Viga sometida a una fuerza puntual $F$ en el extremo libre}

En este caso basta considerar $P=0$ en la ecuación (20), con lo que se obtiene la siguiente ecuación diferencial de la elástica:

$$
\frac{d^{2} z}{d x^{2}}=\left(\frac{F}{E I_{n}}\right)^{n}(L-x)^{n}
$$

que puede integrarse de forma inmediata teniendo en cuenta las condiciones de contorno en el empotramiento $x=0$ (ecuaciones (22) y (23)), obteniéndose la siguiente expresión para la elástica de la viga en voladizo:

$$
z=\frac{1}{(n+1)(n+2)}\left(\frac{F}{E I_{n}}\right)^{n}\left[(L-x)^{n+2}+(n+2) L^{n+1} x-L^{n+2}\right]
$$

Esta expresión permite calcular el desplazamiento vertical $z$ de cada sección de la viga situada a una distancia $x$ del empotramiento. Para la viga en voladizo la flecha $\delta$ se presenta en el extremo libre y su valor se obtiene sustituyendo $x=L$ en la ecuación (25):

$$
\delta=\frac{L^{n+2}}{n+2}\left(\frac{F}{E I_{n}}\right)^{n}
$$

Es importante señalar que la ecuación de la elástica (25) es válida siempre y cuando el cuadrado de la pendiente de la elástica sea mucho menor que la unidad, pues esa ha sido la hipótesis para obtener la ecuación (12), es decir, debe cumplirse la condición:

$$
\left(\frac{d z}{d x}\right)^{2}<<\quad 1
$$

A la hora de analizar un problema particular es necesario comprobar en primer lugar si la aproximación de pequeñas pendientes es aplicable. Para ello basta con derivar la ecuación (25) respecto a la variable $x$ para obtener la pendiente de la elástica en cada punto:

$$
\frac{d z}{d x}=\frac{1}{n+1}\left(\frac{F}{E I_{n}}\right)^{n}\left[L^{n+1}-(L-x)^{n+1}\right]
$$

evaluarla en el extremo libre de la viga $(x=L)$, pues en este punto se produce la máxima pendiente de la elástica $\left(\operatorname{tg} \varphi_{0}\right)$ :

$$
\operatorname{tg} \varphi_{0}=\left(\frac{d z}{d x}\right)_{x=L}=\frac{L^{n+1}}{n+1}\left(\frac{F}{E I_{n}}\right)^{n}
$$

y comprobar que su cuadrado es mucho menor que la unidad. 
Por otra parte, para el caso particular en el que el material es elástico lineal, es decir, $n=1$, las ecuaciones (25) y (26) se transforman en:

$$
\begin{gathered}
z=\frac{F}{E I_{1}}\left(\frac{L x^{2}}{2}-\frac{x^{3}}{6}\right) \\
\delta=\frac{F L^{3}}{3 E I_{1}}
\end{gathered}
$$

que son las que aparecen en la bibliografía [11].

Resulta interesante introducir el parámetro adimensional $\xi$ definido como:

$$
\xi=L^{n+1}\left(\frac{F}{E I_{n}}\right)^{n}
$$

que permite escribir las ecuaciones (26) y (29) en la forma más sencilla:

$$
\begin{gathered}
\frac{\delta}{L}=\frac{\xi}{n+2} \\
\operatorname{tg} \varphi_{0}=\frac{\xi}{n+1}
\end{gathered}
$$

Si la condición de validez de la aproximación para pequeñas pendientes (ecuación (20)) la suponemos válida si el cuadrado de la pendiente máxima es menor, por ejemplo, del $10 \%$ de la unidad, lo que implica $t g^{2} \varphi_{0}<0.1$, entonces de la ecuación (34) se obtiene la siguiente condición de validez de esta aproximación:

$$
\xi<\frac{n+1}{\sqrt{10}}
$$

Por ejemplo, para cobre endurecido $(E=459 \mathrm{MPa}$ y $n=2.16$ ) esta condición indica que la aproximación para pequeñas pendientes es válida para $\xi<1$. En las Figs 5 y 6 se han representado, para cobre endurecido, los valores de $\varphi_{0}$ y $\delta / L$ frente al parámetro $\xi$ calculados mediante las ecuaciones (33) y (34) para la zona de validez de la aproximación considerada en este trabajo (hasta $\xi=1$ para este material).

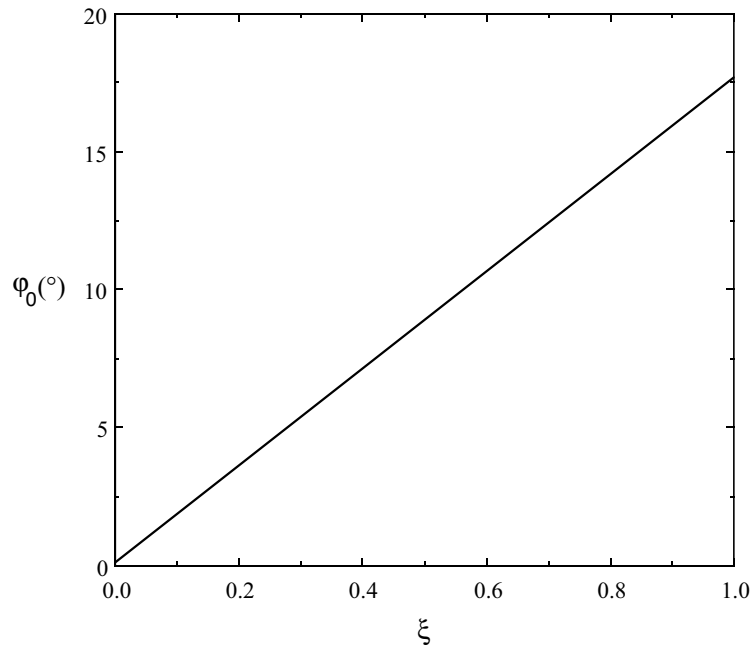

Figura 5. Valores de $\varphi_{0}$ frente a $\xi$ calculados mediante la aproximación para pequeñas pendientes para el caso del cobre endurecido ( $E=459 \mathrm{MPa}$ y $n=2.16)$.

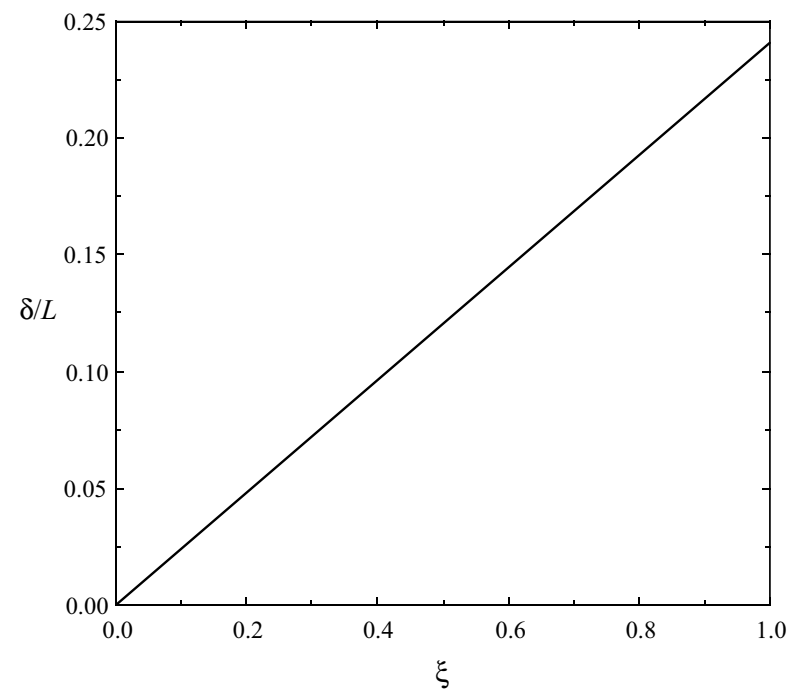

Figura 6. Valores de $\delta / L$ frente a $\xi$ calculados mediante la aproximación para pequeñas pendientes para el caso del cobre endurecido $(E=459 \mathrm{MPa}$ y $n=2.16)$.

\section{III.2. Viga sometida a una fuerza distribuida $\mathbf{P}$ a lo largo de su longitud}

Ahora basta poner $F=0$ en la ecuación (20), con lo que se obtiene la ecuación diferencial:

$$
\frac{d^{2} z}{d x^{2}}=\left(\frac{P}{2 L E I_{n}}\right)^{n}(L-x)^{2 n}
$$

integrando como en el caso anterior, se obtiene la ecuación de la elástica:

$$
\begin{gathered}
z=\frac{1}{(n+1)(2 n+1)}\left(\frac{P}{2 L E I_{n}}\right)^{n} \times \\
{\left[-L^{2 n+2}+L^{2}(L-x)^{2 n}+2 L^{2 n+1}(n+1) x-2 L(L-x)^{2 n} x+(L-x)^{2 n} x^{2}\right]}
\end{gathered}
$$


y sustituyendo $x=L$ en la ecuación (37) se puede calcular la flecha a partir de la expresión:

$$
\delta=\frac{L^{n+2}}{2^{n+1}(n+1)}\left(\frac{P}{E I_{n}}\right)^{n}
$$

que para $n=1$ corresponden al caso de comportamiento lineal. El valor de la flecha para $n=1$ es el que aparece en la bibliografía para el caso lineal [6,9]:

$$
\delta=\frac{P L^{3}}{8 E I_{1}}
$$

\section{III.3. Viga sometida a una fuerza puntual F y a una fuerza distribuida $P<<F$}

Si la fuerza distribuida $P$ (que puede ser, por ejemplo, el peso propio de la viga) es mucho menor que la fuerza puntual aplicada $F$, podemos realizar la siguiente aproximación:

$$
\left[1+\frac{P}{2 F L}(x-L)\right]^{n} \approx 1+\frac{n P}{2 F L}(L-x)
$$

que sustituida en la ecuación (20), proporciona la siguiente ecuación diferencial de la curva elástica:

$$
\frac{d^{2} z}{d x^{2}}=\left(\frac{F}{E I_{n}}\right)^{n}(L-x)^{n}\left[1+\frac{n P}{2 F L}(x-L)\right]
$$

cuya integración es inmediata, aunque el resultado es algo engorroso. Por esta razón únicamente incluimos el valor de la flecha, es decir, del desplazamiento del extremo libre de la viga:

$$
\delta=\frac{L^{n+2}[2(n+3) F+n(n+2) P]}{2(n+2)(n+3) F}\left(\frac{F}{E I_{n}}\right)^{n}
$$

que para $n=1$ coincide con la flecha para el caso de comportamiento lineal del material y no es otra cosa que la suma de las flechas de las ecuaciones (31) y (39):

$$
\delta=\frac{L^{3}}{24 E I_{1}}(8 F+3 P)
$$

\section{Conclusiones}

Partiendo de la hipótesis de que el material del que está fabricada una viga flexionada es elástico no lineal de tipo Ludwick, se ha obtenido la ecuación diferencial de la elástica de la viga de forma análoga a como se hace en el libro de Feynman. Esta ecuación diferencial se ha simplificado considerando el caso de pequeños desplazamientos de la directriz de la viga (pendientes pequeñas) y se ha resuelto para el caso de una viga empotrada en un extremo sometida a distintos tipos de cargas. Se han obtenido las ecuaciones de la elástica y la flecha, comparándolas con las correspondientes al caso de material elástico lineal. Este estudio permite presentar a los estudiantes que el comportamiento elástico de los materiales no es siempre lineal y que los resultados que se obtienen son diferentes dependiendo de cómo sea este comportamiento. Por último, en el desarrollo se han utilizado conceptos físicos de gran interés en un curso de Mecánica (esfuerzo, momento flector, fuerza puntual, fuerza distribuida, elástica, momento de inercia de una sección plana, flexión, deformación, etc.), así como de cálculo infinitesimal (derivada, integral, radio de curvatura de una curva, etc.).

\section{Referências}

[1] W. F. Riley y L. D. Sturges, Ingeniería Mecánica: Estática (Reverté, Barcelona, 1995).

[2] F. Belmar, A. Garmendía y J. Linares, Curso de Física Aplicada: Estática (Universidad Politécnica de Valencia, 1987)

[3] M. R. Ortega, Lecciones de Física: Mecánica 3 (Edita el autor, Córdoba, 1987).

[4] A. Bedford y W. Fowler, Mecánica para Ingeniería: Estática (Addison-Wesley Iberoamericana, Wilmington, 1987)

[5] L. Ortiz-Berrocal, Resistencia de Materiales (McGrawHill, Madrid, 1997).

[6] R. C. Hibbeler, Mecánica de Materiales (Prentice Hall, México, 1998).

[7] A. Beléndez, C. Neipp y T. Beléndez, "Estudio experimental de una viga en voladizo", Rev. Esp. Fis. 15 (3) 42-45 (2001).

[8] F. R. Zypman y C. Guerra-Vela, "The macroscopic scanning force 'microscope"', Eur. J. Phys. 22, 17-30 (2001).

[9] Th. Höpfl, D. Sander y J. Kirschener, "Demonstration of different bending profiles of a cantilever caused by a torque or a force", Am. J. Phys. 69, 1113-1115 (2001).

[10] T. Beléndez, C. Neipp y A. Beléndez, "Large and small deflections of a cantilever beam", Eur. J. Phys. 23, 371-379 (2002).

[11] R. Feynman, R. B. Leighton y M. Sands, Física, Vol. II: Electromagnetismo y Materia (Addison-Wesley Iberoamericana, México, 1987).

[12] M. Sathyamoorthy, Nonlinear Analysis of Structures (CRC Press, Boca Raton, 1998).

[13] K. Lee, "Large deflections of cantilever beams of nonlinear elastic material under a combined loading", Int. J. Non-linear Mech. 37, 439-443 (2002). 